Marquette University

e-Publications@Marquette

Biomedical Engineering Faculty Research and Publications

7-15-2009

\title{
Discriminating Pulmonary Hypertension Caused by Monocrotaline Toxicity from Chronic Hypoxia by Near-Infrared Spectroscopy and Multivariate Methods of Analysis
}

Simon Duri

Marquette University, simon.duri@marquette.edu

Robert C. Molthen

Marquette University, robert.molthen@marquette.edu

Chieu D. Tran

Marquette University, chieu.tran@marquette.edu

Follow this and additional works at: https://epublications.marquette.edu/bioengin_fac

Part of the Biomedical Engineering and Bioengineering Commons

\section{Recommended Citation}

Duri, Simon; Molthen, Robert C.; and Tran, Chieu D., "Discriminating Pulmonary Hypertension Caused by Monocrotaline Toxicity from Chronic Hypoxia by Near-Infrared Spectroscopy and Multivariate Methods of Analysis" (2009). Biomedical Engineering Faculty Research and Publications. 395.

https://epublications.marquette.edu/bioengin_fac/395 
Marquette University

e-Publications@Marquette

\section{Biomedical Engineering Faculty Research and Publications/College of} Engineering

This paper is NOT THE PUBLISHED VERSION; but the author's final, peer-reviewed manuscript. The published version may be accessed by following the link in the citation below.

Analytical Biochemistry, Vol. 390, No. 2 (July 15, 2009): 155-164. DOI. This article is (C) Elsevier and permission has been granted for this version to appear in e-Publications@Marquette. Elsevier does not grant permission for this article to be further copied/distributed or hosted elsewhere without the express permission from Elsevier.

\section{Discriminating Pulmonary Hypertension Caused by Monocrotaline Toxicity from Chronic Hypoxia by Near-Infrared Spectroscopy and Multivariate Methods of Analysis}

Simon Duri

Department of Chemistry, Marquette University, Milwaukee, WI

Robert C. Molthen

Department of Chemistry, Marquette University, Milwaukee, WI

Chieu D.Tran

Department of Chemistry, Marquette University, Milwaukee, WI 53201, USA 


\section{Abstract}

A new method has been developed for the determination of tissue pathology caused by chronic hypoxia and monocrotaline toxicity. The method is based on the use of near-infrared (NIR) spectrophotometry to measure spectra of lung tissue from normal chronic hypoxia $(\mathrm{CH})$ and monocrotaline (MCT) models of pulmonary hypertension (PH), followed by analysis using multivariate methods, that is, principal component analysis (PCA) and partial least squares (PLS). Synergistic use of NIR with the PCA/PLS method makes it possible, for the first time, not only to divide different lung tissue samples into their respective groups (normal, $\mathrm{CH}$, and $\mathrm{MCT}$ ) but also to gain insight into mechanisms of PH caused by $\mathrm{CH}$ and $\mathrm{MCT}$ toxicity. Specifically, $\mathrm{MCT}$ metabolites and other hypertensive conditions are known to produce subtle and minor chemical changes in the compositions of tissue (e.g., proteins, carbohydrates, lipids). Although these changes were detected by the NIR technique, they were too small to be discerned through visual inspection of the spectra. However, they can be accurately classified and properly assigned by the PCA/PLS method. The fact that different tissue types can be accurately divided into their corresponding groups by the NIR and PCA/PLS methods suggests that chemical alterations and mechanisms of pulmonary vascular remodeling and $\mathrm{PH}$ induced by MCT are different from those induced by $\mathrm{CH}$.

\section{Keywords}

Pulmonary hypertension, Monocrotaline toxicity, Near-infrared, Multivariate methods

Monocrotaline (MCT) ${ }^{2}$ is an 11-membered macrocyclic diester member of the pyrrolizidine alkaloid family of plant toxins that produces a delayed onset yet progressive pulmonary vascular disease resulting in pulmonary hypertension (PH) in Sprague-Dawley rats [1], [2], [3]. Therefore, MCT-induced PH has been used as a model for the study of chronic pulmonary vascular diseases in humans since 1961 [4]. Nevertheless, the exact mechanism by which MCT causes pulmonary toxicity is still not completely understood [3]. Considerable efforts have been made by many research groups to elucidate the mechanism [5], [6], [7], [8]. Some studies have shown monocrotaline pyrroles to react with DNA and other cell macromolecules [9], [10], [11], [12]. Unfortunately, to date, only limited success has been made. This is probably due to the lack of a technique that can noninvasively detect and identify MCT and all of its intermediates and products during its biochemical transformation processes. Near-infrared (NIR) multispectral imaging technique may be the solution to this problem.

We have succeeded in developing a novel NIR multispectral imaging (NIR-MSI) instrument that can provide, for the first time, the means to noninvasively, sensitively (single pixel resolution), and rapidly (ms) record not at a single wavelength as in NIR bioimaging instruments but rather on an entire NIR absorption spectrum (as with a Fourier transform [FT]-IR spectrophotometer) and not just one spectrum but rather tens of thousands of spectra at tens of thousands of different locations within a sample during a period as short as a few milliseconds [13], [14], [15], [16], [17]. Chemical information derived from these spectra will make it possible to accurately detect and identify any different chemical or biochemical species at many different positions within a sample. Because of its advantages, we were able to use the NIR-MSI instrument for studies and measurements that are not possible otherwise. These include the noninvasive and nondestructive authentication of documents (e.g., stock certificates, currency), determination of the chemical inhomogeneity of polymers, kinetics of fast reactions (e.g., curing of epoxy by amine), and detection of combinatorial peptide synthesis [13], [14], [15], [16], [17]. It is expected that combined advantages of NIR light (deep tissue penetration) and MSI technique (ability to provide chemical composition and structure at various positions within a sample) enable the NIR-MSI instrument to be uniquely suited for the noninvasive investigation of MCT toxicity in the lung of Sprague-Dawley rats. The anticipation stems from the fact that the NIR-MSI technique 
should be able to detect and identify MCT directly on the lung, follow its biochemical transformation process, and identify all intermediates and products.

Our objective is to use this NIR-MSI instrument to investigate the mechanism of MCT toxicity. To approach this goal, in this preliminary study, we determined whether it is in fact possible to use the NIR spectrophotometric method to chemically detect and identify, directly on lung tissue, not only MCT but also any alterations on lung tissue caused by MCT. In this article, we report, for the first time, our integrated approach to synergistically combine NIR measurements with multivariate methods of analysis to chemically detect and identify normal and diseased rat lung tissue.

\section{Materials and methods}

Samples used were histological slides prepared from lung tissue of rats (Sprague-Dawley and fawn-hooded). One set of animals was left under normal conditions, another was subjected to chronic hypoxia ( $\mathrm{CH}$ ), and the remaining set was given a single subcutaneous injection of $\mathrm{MCT}(60 \mathrm{mg} / \mathrm{kg})$ and raised in room air. The $\mathrm{CH}$ exposure was achieved by housing the animals in chambers maintained at $10 \% \mathrm{O}_{2}$. $\mathrm{CH}$ is also known to cause $\mathrm{PH}$, but through significantly different mechanisms than MCT exposure.

All rats also had partial left pulmonary artery occlusion (PLPAO) surgery in which the left pulmonary artery is banded at a fixed diameter approximately one-third of its normal value. This had the effect of reducing blood flow to the left lung and increasing blood flow to the right lung. In one set of animals PLPAO surgery was done 3 days after exposure to MCT, and in another set it was done 3 days before MCT exposure. All of the studies were done under the approval of the Zablocki VA Medical Center's institutional animal care and use committee review board and in compliance with the National Research Council's guide for the care and use of laboratory animals.

After 24 days, the rats were anesthetized with sodium pentobarbital ( $40 \mathrm{mg} / \mathrm{kg}$, ip for intraperitoneal) and heparinized $(200 \mathrm{lU} / \mathrm{kg}$ ) through right ventricular injection, and the lungs were excised, distended, and fixed with a $10 \%$ buffered formalin solution. The lungs were then paraffin-embedded, and 4- $\mu \mathrm{m}$-thick sections were prepared on slides and stained with hematoxylin and eosin. The slides were measured without any further treatment. NIR spectra were taken on the home-built NIR spectrometer based on an acousto-optic tunable filter. This NIR spectrometer was described in detail in our previous articles [18], [19], [20]. Unless otherwise stated, each spectrum at a single position in a slide was an average of 2000 spectra taken at a 2-nm interval from 1300 to $2100 \mathrm{~nm}$. The absorption of each sample was measured at six different locations. This not only increased the sample size but also facilitated determination of variation within a sample. Information on spatial distribution of $\mathrm{PH}$ in the sample tissues could be obtained by this procedure.

A total of 180 spectra were obtained for analysis of the right lung. Each of the three groups (normal right lung, $\mathrm{CH}$ right lung, and $\mathrm{MCT}$ right lung) had 60 spectra. Of the 60 spectra for each group, we used 54 spectra to construct the calibration model, with the remaining 6 spectra being used for prediction. The left-side samples had 20 spectra for each group. Of the 20 spectra for each group, we used 16 spectra for calibration, leaving 4 samples for prediction. Because there was no independent validation set in this analysis, the cross-validation method was used. Multivariate analysis was done on the raw spectra without any preprocessing using Unscrambler (version 9.2, Camo ASA), similar to the procedures used in our previous publications [18], [19], [20].

\section{Results and discussion}

Examples of the NIR spectra for normal, $\mathrm{CH}$-treated, and MCT-treated lung tissue are shown together in Fig. 1. In this NIR region (1300-2100 $\mathrm{nm}$ or $\left.4700-7700 \mathrm{~cm}^{-1}\right)$, the absorption is relatively small and the spectra for the 
three different samples are quite similar. As illustrated, it would be difficult to distinguish these three samples just by visibly inspecting their NIR spectra. However, it is important to note that even though the NIR spectra of the samples are very similar, they are not identical. Small differences do exist among them, and these small differences can, in principle, be appropriately analyzed using multivariate methods of analysis such as principal component analysis (PCA) and partial least squares (PLS). To test this possibility, we used different PCA/PLSbased methods to analyze NIR spectra so as to determine whether any of these multivariate methods are effective in identifying normal and pathological ( $\mathrm{CH}$ and $\mathrm{MCT}$ ) lung tissue. Results obtained are described in following sections.

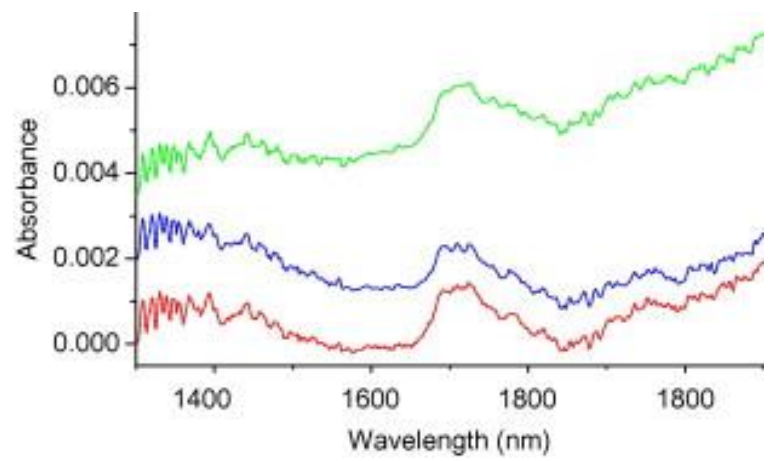

Fig. 1. NIR spectra of normal (blue), $\mathrm{CH}$-treated (green), and MCT-treated (red) lung tissues. (For interpretation of the references to color in this figure legend, the reader is referred to the web version of this article.)

\section{Principal component analysis}

PCA was used to determine whether there is any systematic variation in different lung tissue sample types based on their NIR spectral data. The first task was to study the variation between the different groups, namely how each sample group is different from one another. Therefore, a single PCA model was constructed from a total of 108 NIR spectra, that is, 36 from each sample type (normal, $\mathrm{CH}$, and MCT). All of the sample types were from the right side of the lung. Fig. $2 \mathrm{~A}$ shows the three-dimensional scores plot obtained from this model for the first three principal components (PCs 1,2, and 3). Because scores plots describe the data structure in terms of sample patterns, they can show differences and similarities among samples [21]. As illustrated, the samples are not randomly scattered but rather systematically fall into three nearly distinct groups corresponding to the sample types. The normal samples are relatively well separated from the other two groups. This distinction is clearly illustrated in Fig. 2B, which is a line plot of the sample scores along PC 3. Along this PC, most normal samples have positive scores, whereas the $\mathrm{CH}$ and $\mathrm{MCT}$ samples have negative scores. This clearly indicates that PCA can distinguish normal samples from PH samples. However, some degree of overlap was observed between the $\mathrm{CH}$ and $\mathrm{MCT}$ samples. This is not unexpected given that both of these sample types are from rat lungs with increased vascular remodeling and perfusion resistance (i.e., PH). The results clearly demonstrate that different lung tissue samples can be classified into their respective groups by using the PCA method to analyze their NIR spectra. 


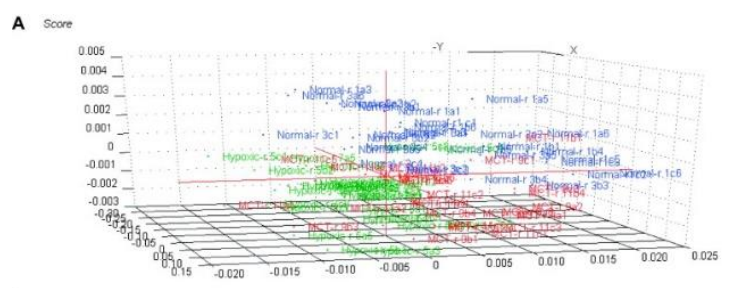

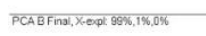

B

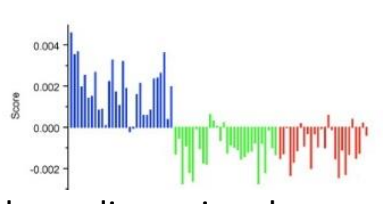

Fig. 2. (A) PCA three-dimensional scores plot. (B) Line plot for the three sample types shown for PC 3. In both graphs, normal samples are in blue, $\mathrm{CH}$ samples are in green, and $\mathrm{MCT}$ samples are in red. (For interpretation of the references to color in this figure legend, the reader is referred to the Web version of this article.)

After it was shown that the NIR spectral data can be used to distinguish sample types, PCA loadings were then used to gain insight into the variables (i.e., spectral range) that are contributing most to the observed differences. To accomplish this, we applied PCA on the data matrix of each group separately. This allows the comparison of the PC loading spectra of the different sample types, in turn making it possible to determine how, and in which spectral range, they are different. Loadings describe the data structure in terms of variable correlations. Each variable has a loading on each PC, and this reflects how much the variable contributes to that PC. The loadings for the first three components are shown in Fig. 3. The loading spectra show that the greatest variation in the loadings for the different samples occur in the region from 1600 to $1800 \mathrm{~nm}$. This could be the most important spectral region in describing the variation of the three sample types. There is no single PC where the loadings for the three sample types are different. However, the $\mathrm{CH}$ loading spectrum is clearly different from the other two sample types for PC 2, whereas the MCT loading spectrum is clearly different from the others for PC 3. These results indicate that $\mathrm{CH}$ produced some alterations in the lungs that can be best represented by PC 2, whereas the alterations of MCT are best represented by PC 3. The results also seem to imply that the two conditions, $\mathrm{CH}$ and $\mathrm{MCT}$ treatment, produce somewhat different chemical transformations in the tissue samples. However, the exact nature of these different chemical alterations cannot be determined with the current set of rather limited data collected in this study. 

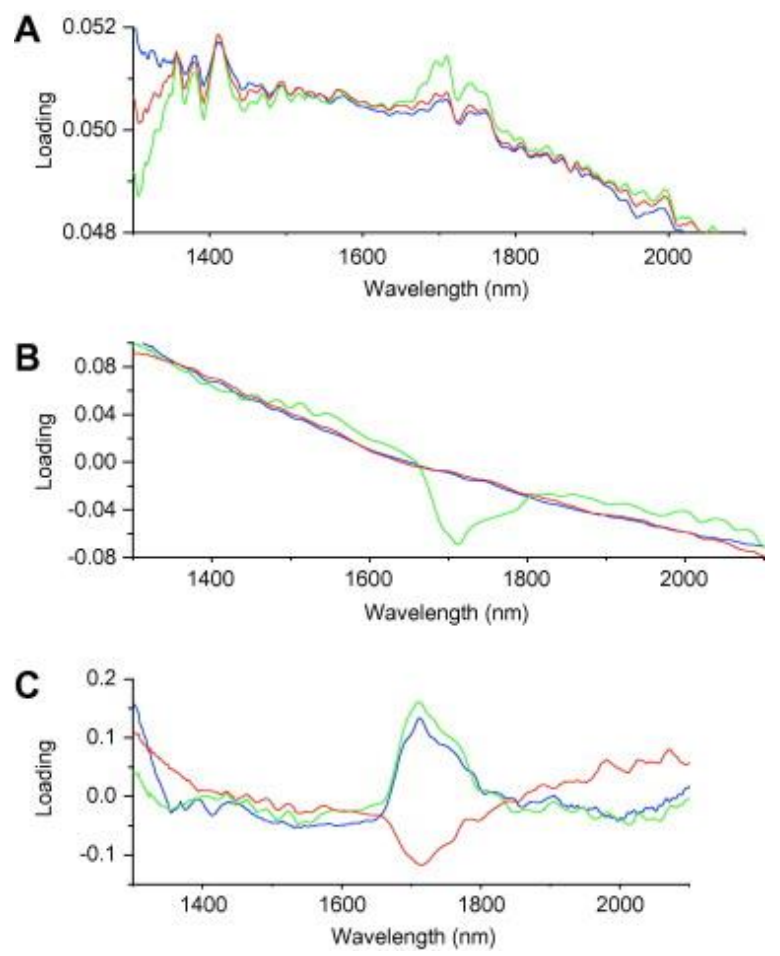

Fig. 3. PCA loading spectra for PC 1 (A), PC 2 (B), and PC 3 (C). Normal, $C H$, and $M C T$ samples are in blue, green, and red, respectively. (For interpretation of the references to color in this figure legend, the reader is referred to the Web version of this article.)

In addition to the aforementioned PCA method, we also used the PLS method in this study. Although both methods are designed to extract useful information from NIR spectra of lung tissue samples, they are not the same, with each method having its own advantages. Specifically, the PCA method is performed on the $x$ data matrix without looking at the correlation between the $x$ and $y$ variables. Therefore, it is best at studying sample patterns and correlations between samples and variables. This is achieved by analyzing PCA scores and loadings. In this study, both PCA and PLS scores plots were used to study sample patterns. PCA loading spectra were used to identify the spectral regions that contributed most to the differences in normal, hypoxic, and MCT sample types.

PLS, on the other hand, models both the $x$ and $y$ data matrices simultaneously to find the latent variables in $x$ that will best predict the latent variables in $y$. Therefore, PLS is a method of constructing predictive models when the goal is not necessarily trying to understand the underlying relationships between samples and variables, or just among variables, but rather just trying to make prediction of the $y$ variables from the $x$ variables. Because PLS is essentially a regression technique, it was used for predicting unknown sample sets into their respective groups. The information obtained from PCA and PLS was highly complementary, with PCA being highly useful in understanding the sample-variable relationships among normal, hypoxic, and MCT sample groups and PLS being useful in predicting unknown samples into their respective groups. Results obtained using PLS methods are presented in the following sections.

\section{Analysis by PLS regression}

Fig. 4A shows the residual validation variance plot for the NIR spectra of the samples obtained using a PLS/LDA (linear discrimination analysis) method. This technique essentially classifies the samples into their respective groups before performing the PLS model. Samples are classified by assigning them a value of 1 for the group to which they belong and a value of 0 for the other groups. Because there are three groups, this classification will 
result in a sample having three $y$ values, allowing the use of the PLS 2 method. PLS 2 is different from PLS 1 because at least two $y$ variables are used in the former, whereas only one $y$ variable is used in the latter. Residual validation variance shows the remaining amount of variation that has not been addressed by model PCs. Accordingly, a PC that has the smallest residual variation variance value is the one that best explains the variation in the samples. As illustrated, a relatively large decrease in residual validation variance was found for PCs 2 and 3. This is an indication that these two PCs can be used to account for a great deal of the variation in the samples that were used to make this model. Accordingly, these two PCs together with PC1 were used to construct a scores plot to determine the clustering of the samples. In the scores plot, shown in Fig. 4B, the samples were separated into three clusters, and (as expected) these clusters correspond to the three different lung tissue types (normal, $\mathrm{MCT}$, and $\mathrm{CH}$ ). This is similar to the clusters observed with PCA. Although there is some overlap of a few samples, the fact that the samples were separated into three corresponding clusters clearly indicates that multivariate analysis of NIR spectra is effective in distinguishing the samples according to their experimental groups (normal, $\mathrm{MCT}$, and $\mathrm{CH}$ ).

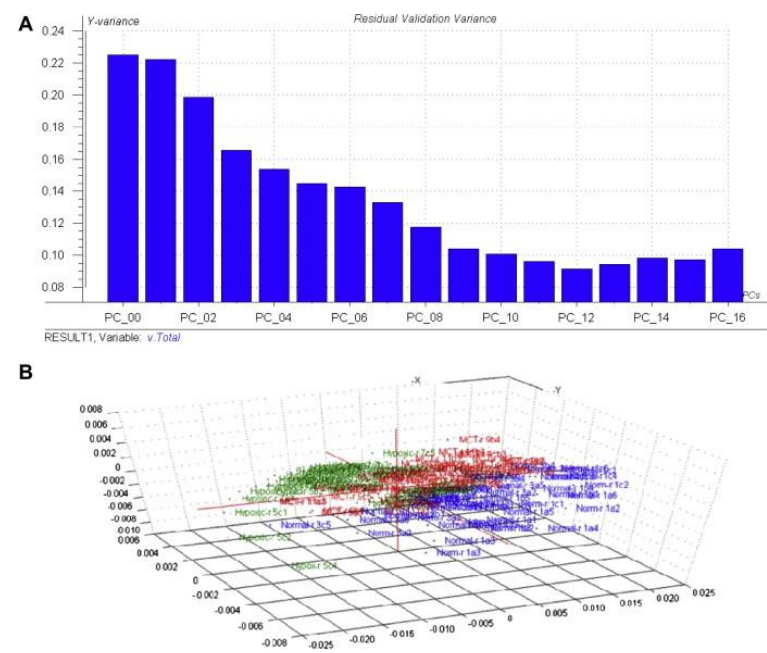

Fig. 4. (A) Residual validation variance plot of NIR spectra. (B) Scores plot obtained using PLS 2 LDA method.

The axes in the above scores plot are PCs 1,2 and 3. It will be informative if we can determine the physical meaning of these PCs. Such information would provide detailed information on differences among different sample types, which in turn would help us to appropriately adjust the experimental procedure to focus on these differences so that the analysis can be improved. Unfortunately, with the spectral information available now, we can only state that each of these PCs represents a single source of independent variation among the samples. It is not possible to determine the physical nature of these variations except to note that the MCT and hypoxia conditions seem to produce alterations in the tissue such that their NIR signatures are different from those of normal cells.

A second set of variables (the $y$ variables) is needed in addition to the spectral data (the $x$ variables) to construct PLS calibration models.

As described above, the objective of this preliminary study was not to mechanistically investigate the PH (this will be the subject of our subsequent studies); rather, it is limited to determining whether a combination of NIR spectrophotometry and multivariate method of analysis can be successfully used to differentiate different tissue types (normal, $\mathrm{CH}$, and $\mathrm{MCT}$ ). To accomplish this, we assigned tissue samples arbitrary values of $\mathrm{PH}$ ranging from 0 to 1 , with 0 being the lowest value of $\mathrm{PH}$ and 1 being the highest. Because the greatest amount of $\mathrm{PH}$ is induced in the right lung lobe of the PLPAO + MCT, all MCT samples were assigned a PH value of 1 . The normal samples were assigned a value of 0 because no $\mathrm{PH}$ is expected in any normal lungs. Cells in $\mathrm{CH}$ lungs, known to 
be hypertensive, are likely to be chemically more similar to MCT samples than to normal samples. Histological studies have also shown that these $\mathrm{CH}$ samples are much more similar to MCT samples than to normal samples. Therefore, they were assigned a $\mathrm{PH}$ value of 0.6. It is noteworthy that there are variations among tissues within each group. However, it is anticipated that these are mainly spatial variations in the chemical composition of the tissues (within each group) and that such spatial variations cannot be determined with spectra obtained with NIR or FT-IR spectrometers that are equipped with single channel detection. They can be determined only by the use of multispectral imaging instrument that essentially is a spectrometer with an area camera as the detector. This will be a subject of our future studies. In the current study, our aim was to determine whether it is possible to divide different lung tissue samples into their respective groups (normal, $\mathrm{CH}$, and $\mathrm{MCT}$ ) by the combined use of NIR spectra and the PCA/PLS method.

The first PLS model was constructed using NIR spectra measured from 1300 to $2100 \mathrm{~nm}$. The calibration set contained 54 samples for each of the three groups (normal, $\mathrm{MCT}$, and $\mathrm{CH}$ ). All of these are from the right lung of the animals. The calibration results from this model are shown Fig. 5, where the black, blue, and red lines represent the target line, the calibration curve, and the validation curve, respectively. The validation method used in this and all the other PLS models discussed below is the full cross-validation method. Cross-validation was used in this case because there was not a sufficient number of samples to make an independent validation set. Cross-validation is performed by calculating a calibration matrix using all of the calibration samples except one. The calibration matrix is then used to predict the concentration of the components in the sample that was left out. The sum-squared errors between the expected and predicted concentrations for this sample are calculated. The procedure is repeated, leaving out a different sample until all of the samples have been computed. In other words, the calculation is done as many times as there are samples [21]. In this program, the validation is done in segments, and the red and blue samples in the figure were samples used for validation and calibration, respectively. As illustrated, the correlation coefficient for the calibration model (blue line) was found to be 0.95 , which is a relatively good correlation considering the fact that those NIR spectra of each slide were measured at six different positions. Similar to results observed with the scores plot, there is some overlap in the predicted values ( $y$ axis) between the $\mathrm{CH}$ and MCT samples. The normal samples are well separated from the rest. In this case, because the variation within each group has not been addressed by this model, the validation correlation coefficient was found to be 0.84 .

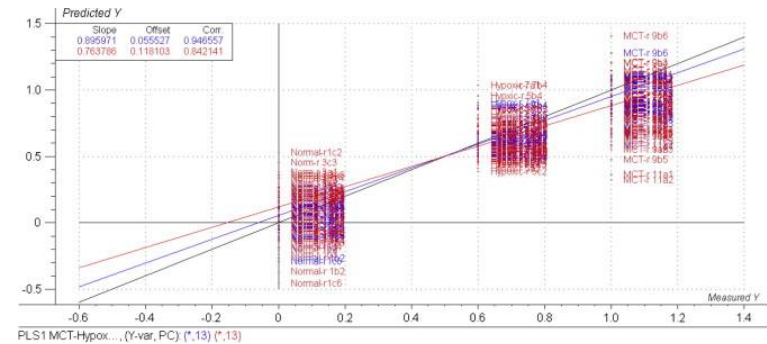

Fig. 5. Predicted versus measured plot for NIR model containing normal, $\mathrm{CH}$, and $\mathrm{MCT}$ right-side samples. The black line is the target line, the blue line is the calibration curve, and the red line is the validation curve. (For interpretation of the references to color in this figure legend, the reader is referred to the Web version of this article.)

PLS 1 was then used for prediction. As described in Materials and Methods, there were 162 samples in the calibration set, that is, 54 from each of the three groups (normal right lung, $\mathrm{CH}$ right lung, and MCT right lung). The unknown set had 18 samples. As shown in Fig. 6A, the residual validation variance of this model decreases up to approximately PC 13. This is the number of components that the model requires to explain all of the systematic variation within the samples. Any latter PCs, whose residual validation variances are now constant or begin to increase, are most likely modeling noise. Accordingly, the prediction was then performed with 13 PCs. 
Although 13 components might seem like a large number, it is appropriate in this case considering the various types of variation in the NIR spectra due to chemical transformation of samples by MCT and $\mathrm{CH}$ treatment. Furthermore, it is significant considering the fact that each spectrum of the samples was measured at 400 individual wavelengths to be able to reduce all spectra of different tissue types to a model with just 13 PCs. It could have been possible to ignore some of the latter PCs suggested by the model and use fewer components for prediction, but it was found that the best prediction is obtained with all of the suggested PCs. Subsequently, the above model was used to predict a set of normal, $\mathrm{CH}$, and MCT samples measured in the same NIR region. The prediction with the deviation is shown in Fig. 6B. Again, in constructing this model, all MCT samples were assigned a $\mathrm{PH}$ value of 1 , normal samples were assigned a $\mathrm{PH}$ value of 0 , and $\mathrm{CH}$ samples were assigned a $\mathrm{PH}$ value of 0.6. As can be seen from Fig. $6 \mathrm{~B}$, all normal samples are predicted to be close to 0 , the $\mathrm{CH}$ samples appear to be around 0.6 , and the MCT samples are around 1.

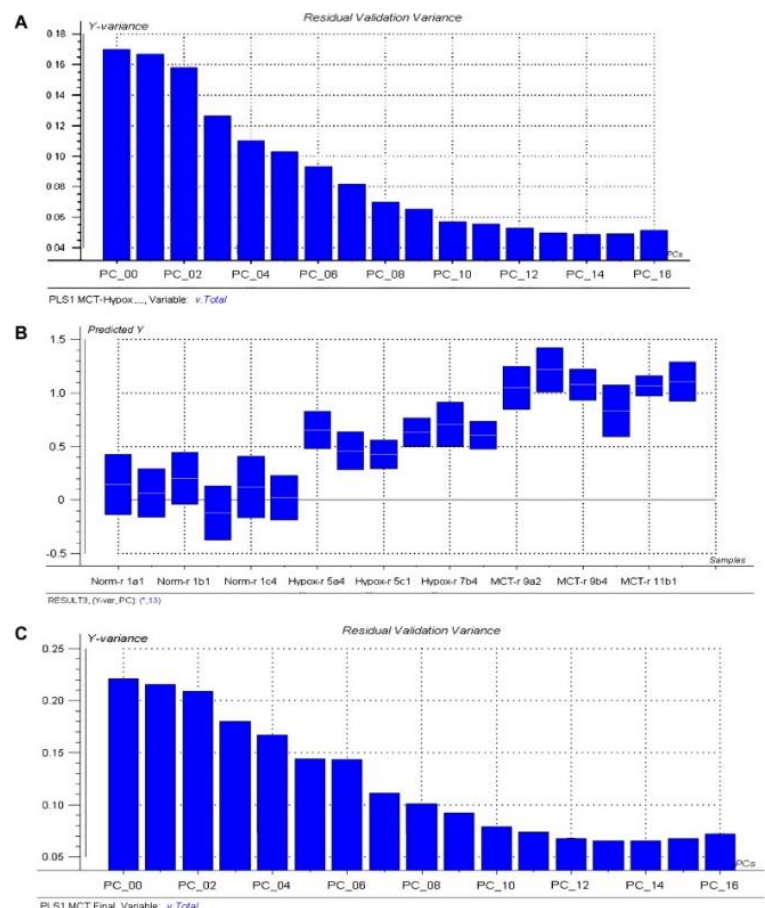

Fig. 6. (A) PLS 1 residual validation variance of the model with NIR spectra. (B) Prediction of unknowns with the model. (C) RMSEP for the model.

Fig. 6 C shows the root mean square error of prediction (RMSEP) for this model. The RMSEP is shown for each component (PC). Just like the residual validation variance, the RMSEP decreases with the components because each successive PC explains less and less variation and, hence, the error also decreases. Just like the residual variance, the error also decreases up to PC 13, which is the number of components the model requires to explain the variation in the samples. The average RMSEP for this model with 13 PCs is 0.296 . The results of Fig. $6 \mathrm{~B}$ clearly indicate that it is possible to distinguish normal cells, $\mathrm{CH}$ cells, and MCT cells using a PLS model based on their NIR spectra. Using this model, it should be possible to correctly predict most of the unknown samples.

The prediction (Fig. 6B) shows that samples within each group do not all appear to be exactly the same. This is consistent with the overlap among the three sample types observed on the scores plot and on the predicted versus measured plots. As explained earlier, this may be due to the fact that chemical alterations in $\mathrm{CH}$ and MCT samples are spatially localized. It seems that not all of the cells in a given type of tissue sample are spatially affected to the same extent. There are some locations within a tissue that remain better (or worse) than others. 
As might be expected, cells in the parenchyma or the veins may be modified less than or differently from cells in the arteries.

\section{Analysis of the left-side samples}

The samples used to construct the model discussed above were made from rats subjected to normoxia, hypoxia, and MCT. In addition, the animals also had PLPAO surgery done on them. This surgery had the effect of chronically reducing blood flow to the left lung while increasing blood flow to the right lung. It has been reported that the right lung, which received increased blood flow, experienced increased structural changes such as neointimal lesions and PH-associated severe pulmonary vascular remodeling (PVREM) [22]. On the other hand, structural changes were reduced in the left lung because of a relatively reduced blood flow.

The PLS method was then used to analyze NIR spectra to determine whether it is possible to distinguish tissues from the right and left lungs. However, it is not possible to use the NIR model as described above to predict the left-side samples given that these samples were not part of the calibration of this model. This is because calibrations for multivariate analysis are required to contain all of the expected components having concentrations and conditions within ranges of interest. Accordingly, new models were constructed using new sets of NIR spectra that contain spectra of the left-side samples. To simplify the analysis, the $\mathrm{CH}$ samples were left out of the new models. Similar to the first models, the normal right-side samples and the MCT right-side samples were assigned $\mathrm{PH}$ values of 0 and 1, respectively. The normal left-side samples were also assigned a PH value of 0 because these animals were not subjected to any hypertensive condition. Because the MCT left-side samples have reduced blood flow and, hence, reduced PVREM, they were assigned an intermediate PH value of 0.5 .

The calibration correlation coefficient for this model was 0.95 , whereas the validation correlation coefficient was 0.84 (Fig. 7B). The average RMSEP for the model was 0.348 , which is higher than that of the previous model that had $\mathrm{CH}$ samples. Careful inspection of the results reveals that, for some reason, the NIR models predict $\mathrm{CH}$ samples relatively better than the MCT samples. Fig. 5 shows that the MCT samples are more scattered than the $\mathrm{CH}$ samples. It could be possible that chemical changes occurring in $\mathrm{CH}$ samples were more readily observable or more spatially homogeneous in the measured NIR region than the changes taking place in the MCT samples.

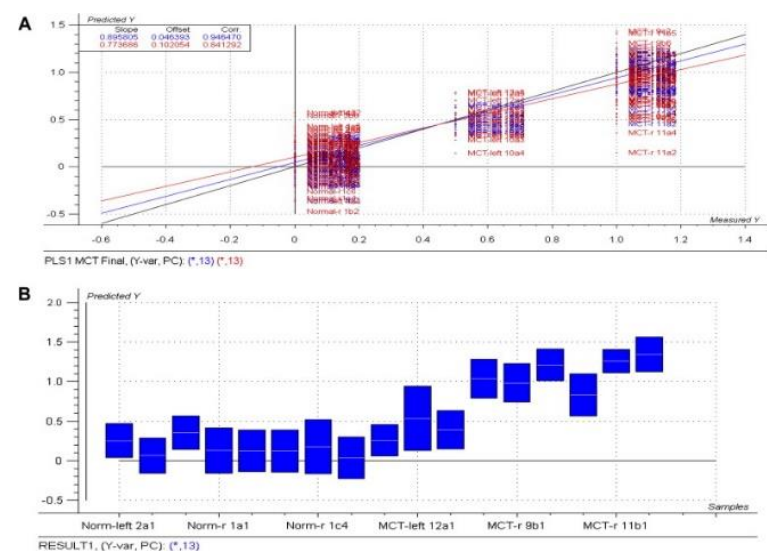

Fig. 7. (A) Predicted with deviation for the MCT left-side NIR model. (B) Prediction of the MCT left-side samples.

However, this NIR model is still able to distinguish the MCT left-side samples from the right-side samples. In Fig. 7C, the MCT left-side samples (samples 9, 10 and 11) are shown as appearing much closer to the normal samples, clearly distinguishing them from the right-side samples. This is consistent with previous studies in which histology from the left lungs of PLPAO + MCT rats is remarkably similar to that from normal lung tissue. The left-side samples still show some signs of pathology because even though the blood flow has been reduced, 
metabolites of MCT still perfuse the left lung given that the blood flow was not completely blocked. This again shows that it is possible to determine the sample types based on their NIR spectra.

\section{Pre- and post-PLPAO samples}

Results obtained so far clearly indicate that a minor change in an animal's condition produces an observable effect on PH on the lung. For example, results obtained on the MCT model based on spectra of left-side samples indicate that, as the result of the decreased blood flow to the left side, the PVREM and PH on this side were reduced. It would be of particular interest to determine whether a minor change in experimental condition may lead to an observable effect on PH. Specifically, the results described so far were based on the use of samples taken from animals on which PLPAO surgery was performed initially, and then 3 days later they were exposed to the different conditions (normal, $\mathrm{CH}$, and $\mathrm{MCT}$ ). If the surgery were performed on the animals not initially but rather a few days after exposure to the different conditions, would this produce any observable change? To investigate this effect, some rats were injected with MCT initially and were subjected to PLPAO surgery on the $3 r d$ day. The experiment was continued as before, and NIR models were created for these samples. Sample types, the pre- and post-PLPAO, were included in the model. Fig. 8A shows the scores plot of the model that includes the pre-PLPAO MCT samples. As illustrated, in the PC 3 direction on the left part of the graph, three sample types were clearly separated: normal samples followed by MCT samples and then $\mathrm{CH}$ samples. This PC seems to contain most of the information related to differences among the three sample types. Although it is not possible to accurately determine the type and origin of these differences at this time, the results presented clearly indicate that it is possible to distinguish normal samples from MCT and $\mathrm{CH}$ samples. Different from the left side, there seem to be relatively greater overlaps among the samples, especially the MCT and $\mathrm{CH}$ samples, on the right side of the map. This may be due to the fact that the $\mathrm{MCT}$ and $\mathrm{CH}$ samples are relatively similar chemically. Again similar to the previous NIR models, the greatest separation is in the direction of PC 3; this seems to imply that it is this PC that carries most of the information that explains the chemical differences found among normal, $\mathrm{CH}$, and MCT samples. It should also be noted that there is no clear systematic separation of the pre-PLPAO MCT samples from the rest of the MCT samples on this scores plot; this seems to suggest that there is no observable difference between pre- and post-PLPAO surgery in the MCT samples, consistent with observations made previously by Okada and coworkers [22].

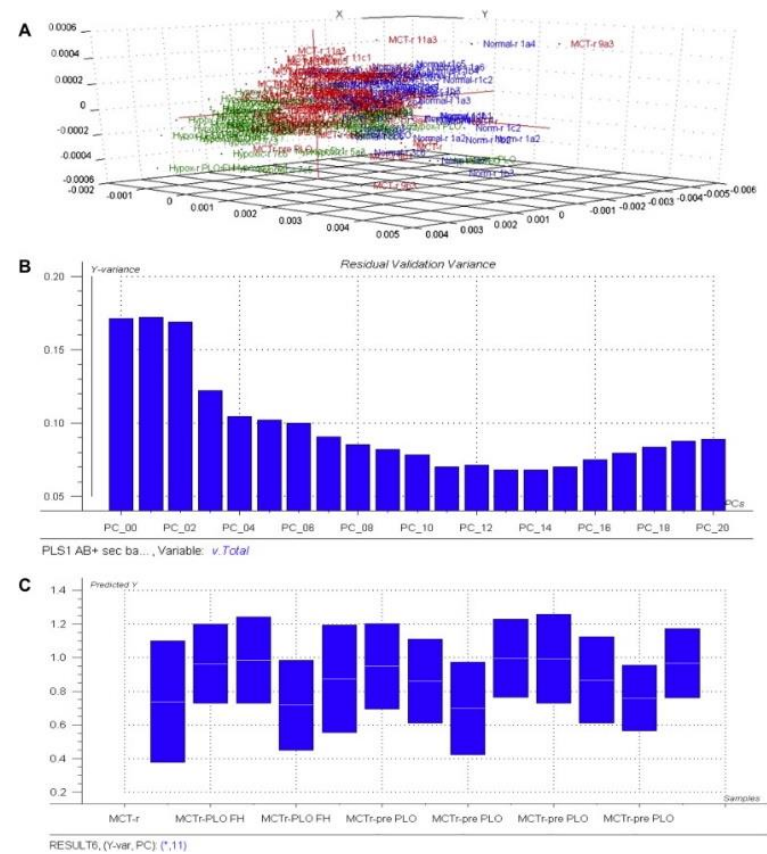

Fig. 8. (A) Scores plot for the model. (B) Residual validation variance plot. (C) Predictions of pre- and post-PLPAO. 
This NIR model had a calibration correlation coefficient of 0.89 , and the validation coefficient was 0.77 . The average RMSEP for this model was 0.232 . This model was then used to predict the pre- and post-PLPAO MCT samples, the results of which are shown in Fig. 8C. As illustrated, most of the samples appear to be close to 1, as is expected of MCT samples. The first five samples in the figure had surgery before exposure to MCT (postPLPAO), and the remainder of the samples had surgery on the 3rd day of exposure (pre-PLPAO). As can be seen, there does not seem to be any significant difference between these samples. These results seem to suggest that pre- and post-PLPAO may lead to the same amount of PVREM and cellular modification, making them chemically indistinguishable. Because four of the post-PLPAO samples are actually from rats of a different species (fawnhooded), it is reassuring to find that the models satisfactorily compensate for genetic variation due to differences in the strain of rats used in the experiments.

\section{Conclusions}

We have demonstrated that the NIR technique can be used not only to determine different tissue types, including normal tissue and tissues damaged by $\mathrm{MCT}$ and $\mathrm{CH}$, but also to gain insight into PH induced by MCT and other experimental conditions. NIR spectra of these tissues are very similar; any differences among them are so small that they cannot be differentiated by simple visual inspection or by univariate methods. However, we showed that by the use of multivariate methods such as PCA and PLS, we can satisfactorily analyze them. For example, normal and pathological lung tissues (i.e., damaged by $\mathrm{CH}$ and $\mathrm{MCT}$ ) can be properly classified by PCA/PLS scores plots.

We successfully showed that the PCA loading spectrum for the $\mathrm{CH}$ tissues was different from that for the other tissue types for PC 2, whereas for the MCT tissues it was found to be PC 3. These results seem to indicate that $\mathrm{CH}$ and $\mathrm{MCT}$ toxicity can lead to different chemical alterations in the cells. Even though the changes in tissues may be very small, they can be readily detected by the combined use of NIR and PCA.

The NIR PLS models were able to divide different lung tissue samples into their respective groups (normal, $\mathrm{CH}$, and MCT). All of the PLS models constructed using the NIR spectra consistently showed relatively large numbers of components. Because components represent sources of variation, it can be concluded that the absorption of tissues in the NIR regions is complex and due to many components. It is known that MCT metabolites and other hypertensive conditions produce chemical changes in the compositions of tissue (e.g., proteins, carbohydrates, lipids, DNA) [8], [22], [23], [24], [25]. Such changes were detected by the NIR technique and classified as components by the PLS method. The fact that different types of tissues can be accurately divided into their corresponding groups (according to their NIR PLS components) seems to suggest that chemical alterations and mechanisms of PVREM and PH induced by MCT are different from those of $\mathrm{CH}$. MCT is believed to undergo metabolism in the liver, where it is converted to toxic metabolites that are then transported to the lungs, where they cause endothelial damage and subsequent PH [5], [6], [7], [8]. Partial occlusion of the left pulmonary artery reduces blood flow to the left lung while increasing blood flow to the right lung. Previous studies showed that such surgery mitigates the PVREM resulting in the left lung and accentuates PVREM in the right lung. Results obtained in the current study using the NIR PLS model indicate that, chemically, the MCT left-side samples are intermediate between normal and MCT right-side samples. This not only is in agreement with findings of previous studies but also indicates that it is indeed possible to use the combined NIR and PLS technique to determine whether a tissue sample came from the lung with reduced or increased blood flow. The fact that NIR PLS results show that the normal left side of lung samples are just like their right-side counterparts seems to indicate that PLPAO itself causes negligible changes to the lungs.

Additional information on the mechanism of MCT toxicity can also be obtained from this study. For example, we found that lung tissue samples from rats that had the order of PLPAO surgery and MCT exposure reversed were indistinguishable. This indicates that the severe PVREM injury develops in the right lung and is absent in the left 
lung, independent of the sequence in which the rat is exposed to MCT or changes in pulmonary blood flow. The fact that results from tissue samples from fawn-hooded rats are similar to those from tissue samples from Sprague-Dawley rats indicates that (i) the NIR PLS method can satisfactorily take into account experimental variation such as strain differences and (ii) animals from different strains are analogously affected by similar challenges.

In summary, we have successfully demonstrated that the NIR technique can be used not only to detect and identify different types of tissues, including normal tissues and tissues damaged by $\mathrm{MCT}$ and $\mathrm{CH}$, but also to gain insight into mechanisms of PH induced by MCT. Due to the fact that an NIR spectrophotometer is based on the use of a single channel detector, it is expected that substantially more detailed information can be obtained if the NIR spectrophotometer is replaced with an NIR MSI instrument. This is because this imaging instrument uses an NIR area camera and can measure not only a single NIR absorption spectrum as the NIR spectrometer but also thousands of spectra simultaneously at thousands of different locations within a sample. Accordingly, we plan to use knowledge gained from this work to guide our next study, which is based on the use of the NIR MSI technique to investigate in vitro and in vivo mechanisms of pulmonary damage caused by MCT toxicity.

\section{References}

[1] E. Valdivia, J. Sonnad, Y. Hayashi, J.J. Lalich. Experimental interstitial pulmonary edema. Angiology, 18 (1967), pp. 378-383

[2] D.W. Wilson, H.J. Segall, L.C. Pan, S.K. Dunston. Progressive inflammatory and structural changes in the pulmonary vasculature of monocrotaline treated rats. Microvasc. Res., 38 (1989), pp. 57-80

[3] D.W. Wilson, H.J. Segall, L.C. Pan, M.W. Lame, J.E. Estep, D. Morrin. Mechanisms and pathology of monocrotaline pulmonary toxicity. Crit. Rev. Toxicol., 22 (1992), pp. 307-325

[4] J.J. Lalich, L. Merkow. Pulmonary arteritis produced in rats by feeding Crotalaria spectabilis. Lab. Invest., 10 (1961), pp. 744-750

[5] A.R. Mattocks. Chemistry and Toxicology of Pyrrolizidine Alkaloids. Academic Press, London (1986)

[6] R.J. Huxtable. Activation and pulmonary toxicity of pyrrolizidine alkaloids. Chem.-Biol. Interact., 76 (1990), pp. 19-30

[7] M.W. Lame, A.D. Jones, D. Morrin, H.J. Segall. Metabolism of $\left[{ }^{14} \mathrm{C}\right]$ monocrotaline by isolated perfused rat liver. Drug Metab. Dispos., 19 (1991), pp. 516-524

[8] L.C. Pan, D.W. Wilson, M.W. Lame, A.D. Jones, H.J. Segall. COR pulmonale is caused by monocrotaline and dehydromonocrotaline, but not by glutathione or cysteine conjugates of dihydropyrrolizine. Toxicol. Appl. Pharmacol., 118 (1993), pp. 87-97

[9] J.R. Hincks, H.Y. Kim, H.J. Segall, R.J. Molyneux, F.R. Stermitz, R.J. Coulombe. DNA cross-linking in mammalian cells by alkaloids: structure-activity relationships. Toxicol. Appl. Pharmacol., 11 (1991), pp. $90-98$

[10] A.R. Mattocks. Toxicity of pyrrolizidine alkaloids. Nature, 271 (1968), pp. 723-728

[11] C.M. Hoorn, R.A. Roth. Monocrotaline pyrrole alters DNA, RNA, and protein synthesis in pulmonary artery endothelial cells. Am. J. Physiol., 262 (1992), pp. L740-L747

[12] H.Y. Kim, F.R. Stermitz, R.J. Coulombe. Pyrrolizidine alkaloid-induced DNA-protein cross-links. Carcinogenesis, 16 (1995), pp. 2691-2697

[13] O. Khait, S. Smirnov, C.D. Tran. Multispectral imaging microscope with millisecond time resolution. Anal. Chem., 73 (2001), pp. 732-739

[14] C.D. Tran, Y. Cui, S. Smirnov. Simultaneous multispectral imaging in the visible and near infrared region: applications in document authentication and determination of chemical inhomogeneity of copolymers. Anal. Chem., 70 (1998), pp. 4701-4708

[15] C.D. Tran. Infrared multispectral imaging: principles and instrumentation. Appl. Spectrosc. Rev., 38 (2003), pp. 133-153 
[16] C.D. Tran. Principles, instrumentation, and applications of infrared multispectral imaging: an overview. Anal. Lett., 38 (2005), pp. 735-752

[17] C.D. Tran, V.I. Grishko, S. Challa. Molecular state and distribution of fullerenes entrapped in sol-gel samples. J. Phys. Chem. B, 112 (2008), pp. 14548-14559

[18] C.D. Tran, V.I. Grishko, D. Oliveira. Determination of enantiomeric compositions of amino acids by nearinfrared spectrometry through complexation with carbohydrate. Anal. Chem., 75 (2003), pp. 64556462

[19] C.D. Tran, D. Oliveira, V.I. Grishko. Determination of enantiomeric compositions of pharmaceutical products by near-infrared spectrometry. Anal. Biochem., 325 (2004), pp. 206-214

[20] C.D. Tran, D. Oliveira, S. Yu. New chiral ionic liquid that functions as both solvent and chiral selector for the determination of enantiomeric compositions of pharmaceutical products. Anal. Chem., 78 (2006), pp. $1349-1356$

[21] R. Kramer. Chemometric Techniques for Quantitative Analysis. Marcel Dekker, New York (1998)

[22] K. Okada, Y. Tanaka, M. Bernstein, W. Zhang, G.A. Patterson, M.D. Botney. Pulmonary hemodynamics modify the rat pulmonary artery response to injury: a neointimal model of pulmonary hypertension. Am. J. Pathol., 151 (1997), pp. 1019-1025

[23] D.W. Tayler, D.W. Wilson, M.W. Lame, S.D. Dunston, A.D. Jones, H.J. Segall. Comparative cytotoxicity of monocrotaline and its metabolites in cultured pulmonary artery endothelial cells. Toxicol. Appl. Pharmacol., 143 (1997), pp. 196-204

[24] H.C. Thomas, M.W. Lame, S.K. Dunston, H.J. Segall, D.W. Wilson. Monocrotaline pyrrole induces apoptosis in pulmonary artery endothelial cells. Toxicol. Appl. Pharmacol., 151 (1998), pp. 236-244

[25] W.M. Lafranconi, R.J. Huxtable. Hepatic metabolism and pulmonary toxicity of monocrotaline using isolated perused liver and lung. Biochem. Pharmacol., 33 (1984), pp. 2479-2484

${ }^{1}$ Permanent address: Division of Pulmonary and Critical Care, Department of Medicine, Medical College of Wisconsin, Milwaukee, WI 53226, USA.

${ }^{2}$ Abbreviations used: $\mathrm{MCT}$, monocrotaline; $\mathrm{PH}$, pulmonary hypertension; NIR, near-infrared; $\mathrm{MSI}$, multispectral imaging; FT, Fourier transform; $\mathrm{CH}$, chronic hypoxia; PLPAO, partial left pulmonary artery occlusion; PCA, principal component analysis; PLS, partial least squares; PC, principal component; LDA, linear discrimination analysis; RMSEP, root mean square error of prediction; PVREM, pulmonary vascular remodeling. 